\title{
Waveform Parameter Evaluation of Lightning Impulse Voltage Based on Neural Network Method
}

\author{
Nutthaphong Tanthanuch, ${ }^{*}$ Savinee Ludpa, ${ }^{2}$ and Peerawut Yutthagowith ${ }^{2}$ \\ ${ }^{1}$ Faculty of Engineering, Thammasat University, \\ 99 Village No. 18 Phahonyothin Road, Klongluang, Rangsit, Pathumthani 12121, Thailand \\ ${ }^{2}$ School of Engineering, King Mongkut's Institute of Technology Ladkrabang, \\ 1 Chalongkrung Road, Ladkrabang, Bangkok 10520, Thailand
}

(Received January 29, 2021; accepted June 2, 2021)

Keywords: lightning impulse test, base curve fitting, waveform parameters, multilayer neural networks, exponential fitting

We propose a new approach based on applying neural networks for the base curve fitting of full lightning impulse voltage waveforms. In the standard IEC 61083-2, the LevenbergMarquardt algorithm is employed in the calculation of the base curve to evaluate the waveform parameters. In this study, multilayer neural networks were constructed to correct the base curve calculated by simple two-exponential fittings. The approach to constructing the networks divides the data into two groups: training data and testing data. The training data were collected from the standard waveforms generated by the test data generator of the standard, where there were 29 cases of full lightning impulse voltage waveforms. The testing data were collected from real experimental data with various overshoot rates. The waveform parameters obtained by the proposed method were compared with those obtained by the standard approach, and very good agreement was always observed. It was found that the proposed method provides very accurate waveform parameters within the acceptable tolerances of the standard. Also, the method has a shorter execution time than the standard method. Therefore, the proposed method is an attractive choice for evaluating lightning impulse voltage waveform parameters.

\section{Introduction}

The failure of insulation in a high voltage (HV) system due to overvoltage from the effects of lightning is one of the major causes of faults in power systems. To confirm the insulation performance of materials used in HV equipment, simulated electrical field stresses in the form of high impulse voltages are applied to the equipment. Therefore, HV impulse testing is essential to confirm the operation of $\mathrm{HV}$ insulation equipment.

Practically, the generated lightning impulse voltage in a test should be in accordance with the standard requirements as shown in Fig. 1. The IEC standard 60060-1 (2010) ${ }^{(1)}$ specifies the test voltage peak according to the system voltage for which the equipment is installed. The tolerance of the peak voltage $\left(U_{p}\right)$ should be in the range of $\pm 3 \%$ of the specified value. The front time $\left(T_{1}\right)$

*Corresponding author: e-mail: tanthanuch1@engr.tu.ac.th https://doi.org/10.18494/SAM.2021.3306 


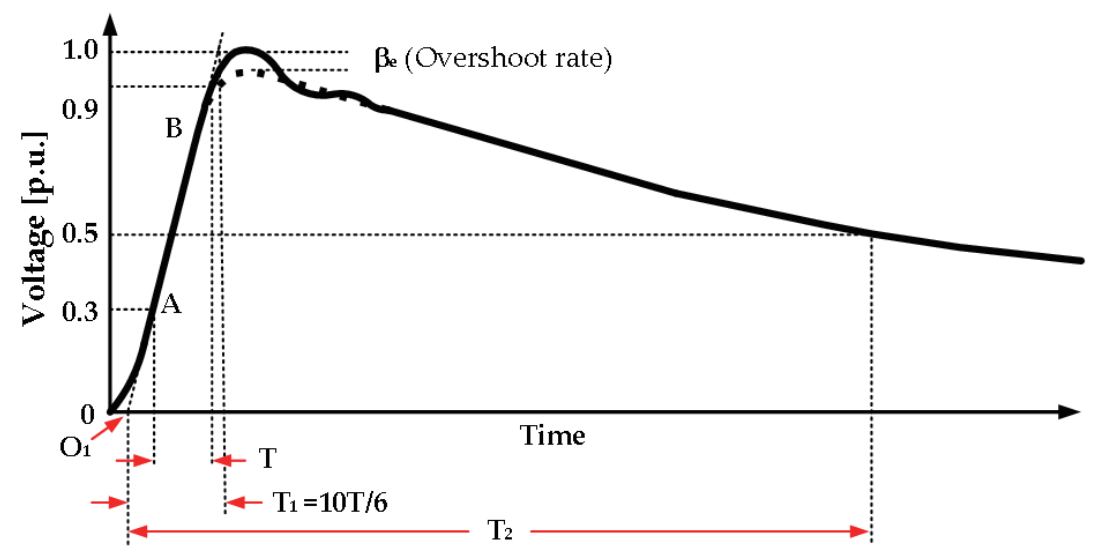

Fig. 1. (Color online) Generated impulse voltage waveform.

and the time to half $\left(T_{2}\right)$ should be $1.2 \mu \mathrm{s} \pm 30 \%$ and $50 \mu \mathrm{s} \pm 30 \%$, respectively. Also, the overshoot rate $\left(\beta_{e}\right)$ should be less than $10 \%$.

For HV measurement, the sensor or the measuring system must scale down the voltage signal to a low level that can be measured by the recording instrument without damage. Also, the measuring system must have a frequency bandwidth covering the frequency range of the lightning impulse voltage from $0 \mathrm{~Hz}$ to several $\mathrm{kHz}$.

In the past, an analog oscilloscope was used to record the voltage waveform, and the waveform parameters were analyzed by the inspection of a test engineer. Later, digital oscilloscopes were used to measure lightning impulse waveforms, and the waveform data were employed to analyze the parameters of the test voltages. Also, the standard ${ }^{(1,2)}$ introduces the software approach based on the construction of a base curve and a $k$-factor filter to evaluate the waveform parameters $\left(U_{p}, T_{1}, T_{2}\right.$, and $\left.\beta_{e}\right)$ as shown in Fig. 2 . There are six procedures employed in the waveform parameter evaluation as follows.

(1) Collect the waveform from $20 \%$ of the peak voltage at the front part to $40 \%$ of the peak voltage at the tail part. This collected waveform is called the recorded waveform $\left[U_{R}(t)\right]$.

(2) Fit the waveform with the function in Eq. (1) provided by the standard. Another form of this function is expressed as Eq. (2), which is utilized to fit the recorded curve waveform. The fitted waveform is called the base curve $\left[U_{b}(t)\right]$.

$$
\begin{gathered}
f(t)=U_{p}\left(e^{-\left(t-t_{d}\right) / \tau_{1}}-e^{-\left(t-t_{d}\right) / \tau_{2}}\right) \\
f(t)=A e^{-\alpha t}+B e^{-\beta t}
\end{gathered}
$$

(3) Subtract the base curve $\left[U_{b}(t)\right]$ from the recorded waveform, and the resulting waveform is called the residual curve.

(4) Filter the residual curve with the $k$-factor filter shown in Eq. (3) to obtain the filtered residual curve. 




Fig. 2. (Color online) Lightning impulse voltage waveforms in parameter evaluation according to the standard.

$$
k(f)=\frac{1}{1+2.2 f^{2}}
$$

Here, $f$ is a frequency in the unit of MHz. According to standard guidelines, the $k$-factor filtering algorithm can be easily implemented using the zero shift phase infinite impulse response (IIR) filter proposed by Lewin et al..$^{(3)}$

(5) Add the filtered residual curve to the base curve to obtain the test waveform $\left[U_{t}(t)\right]$.

(6) The test waveform is used to evaluate the waveform parameters, i.e., the front time $\left(T_{1}\right)$, time to half $\left(T_{2}\right)$, and overshoot rate $\left(\beta_{e}\right)$.

In procedure (2), the Levenberg-Marquardt method has been used to determine the base curve. It was found that the iteration process of the Levenberg-Marquardt method has a long execution time for some waveforms with a high overshoot rate. ${ }^{(3-15)}$ There have been some attempts to overcome this problem. The double integration approach with weight functions ${ }^{(16)}$ and the improved Prony method ${ }^{(17)}$ have been proposed, although complicated calculations are required in these approaches. Therefore, these approaches require a test engineer with experience in software development.

In this article, we propose a method employing a non-iterative curve fitting to evaluate the base waveform parameters of the lightning impulse voltage. The developed method uses a multilayer neural network to determine the base waveform parameters. The training data used in the construction of the network are compiled from the test data generator in the IEC 61083-2 (2013) standard. ${ }^{(18)}$ Some real experimental waveforms are utilized as test cases to confirm the validity of the proposed method in comparison with those calculated by the standard recommended method (Levenberg-Marquardt), and good agreement is observed. The validity of the proposed method has been confirmed in terms of accuracy and execution time, which are superior to those of the standard recommended method owing to absence of iterations.

\section{Proposed Curve Fitting Method}

In the proposed method, a feed-forward multilayer artificial neural network (ANN) ${ }^{(19-21)}$ model was developed to correct the waveform parameters of the base waveform. A backpropagation learning algorithm was utilized in the training process and the construction of the ANN model. The network construction procedures were as follows: 
(1) Prepare the waveform data by separating the recorded waveform $[f(t)]$ into two parts. The first part $\left[f_{1}(t)\right]$ is from $20 \%$ of the peak voltage on the front part to the peak voltage, and the second part $\left[f_{2}(t)\right]$ is from the peak voltage to $40 \%$ of the peak voltage on the tail part.

(2) Fit $f_{2}(t)$ with Eq. (4) to obtain the parameters $A^{\prime}$ and $\alpha^{\prime}$.

$$
g_{1}(t)=A^{\prime} e^{-\alpha^{\prime} t}
$$

(3) Fit $g_{1}(t)-f_{1}(t)$ with Eq. (5) to obtain the parameters $B^{\prime}$ and $\beta^{\prime}$.

$$
g_{2}(t)=B^{\prime} e^{-\beta^{\prime} t}
$$

(4) The estimated base curve can be determined by Eq. (6), which is used as the base curve to calculate the estimated waveform parameters.

$$
g_{3}(t)=A^{\prime} e^{-\alpha^{\prime} t}+B^{\prime} e^{-\beta^{\prime} t}
$$

Then, the estimated waveform parameters, i.e., $A^{\prime}, \alpha^{\prime}, B^{\prime}, \beta^{\prime}$, and $\beta_{e}{ }^{\prime}$ (estimated overshoot rate) can be computed.

(5) The estimated waveform parameters $\left(A^{\prime}, \alpha^{\prime}, B^{\prime}, \beta^{\prime}\right.$, and $\left.\beta_{e}{ }^{\prime}\right)$ are used as the input data, and the network is constructed by a back-propagation algorithm to obtain each parameter $(A, \alpha, B$, and $\beta$ ) of the base curve, which is evaluated by the standard recommended method (Levenberg-Marquardt). Four networks are developed to obtain each base curve parameter. In this paper, 29 waveform data (LI-A1 to LI-A12 and LI-M1 to LI-M17) ${ }^{(18)}$ generated by the test data generator in the standard ${ }^{(18)}$ were utilized as the training data set in the construction process of the neural network. The construction procedures of the multilayer neural networks used in this paper are expressed as the flow chart in Fig. 3. The architecture of the ANNs in this paper is selected to have as low complexity as possible. As shown in Fig. 4, the selected structure of the neural networks comprises a five-neuron input layer, a two-neuron hidden layer, and a oneneuron output layer. The mathematical operations of a neuron in the hidden layer, as shown in Fig. 5, comprise weighting, summation, biasing, and activation with the Tansig function. In the training process to construct the ANN models, some iterations are required as pre-calculation. It takes several seconds to obtain the network. However, the constructed ANN can readily be simplified into matrix form, and the base curve can be determined without further iteration. Therefore, the proposed method is effective in terms of execution time for evaluating the lightning waveform parameters.

Owing to the low complexity of the developed model, it can reduce the model dimension. The weighting and bias constants of the output layer can be included in the weighting and bias constants of the hidden layer. Therefore, the output parameters from the developed model can be computed using Eq. (7), where $x_{i}$ are the input parameters, i.e., $A^{\prime}, B^{\prime}, \alpha^{\prime}, \beta^{\prime}$, and $\beta_{e}{ }^{\prime} . N$ is the number of input parameters, so in this case, $N=5 . y^{(l)}(A, B, \alpha$, or $\beta)$ is a predicted parameter of $(l) . w_{1, i j}^{(l)}$ and $b_{1, i j}^{(l)}$ are the weighting and bias constants of the input layer associated with input 


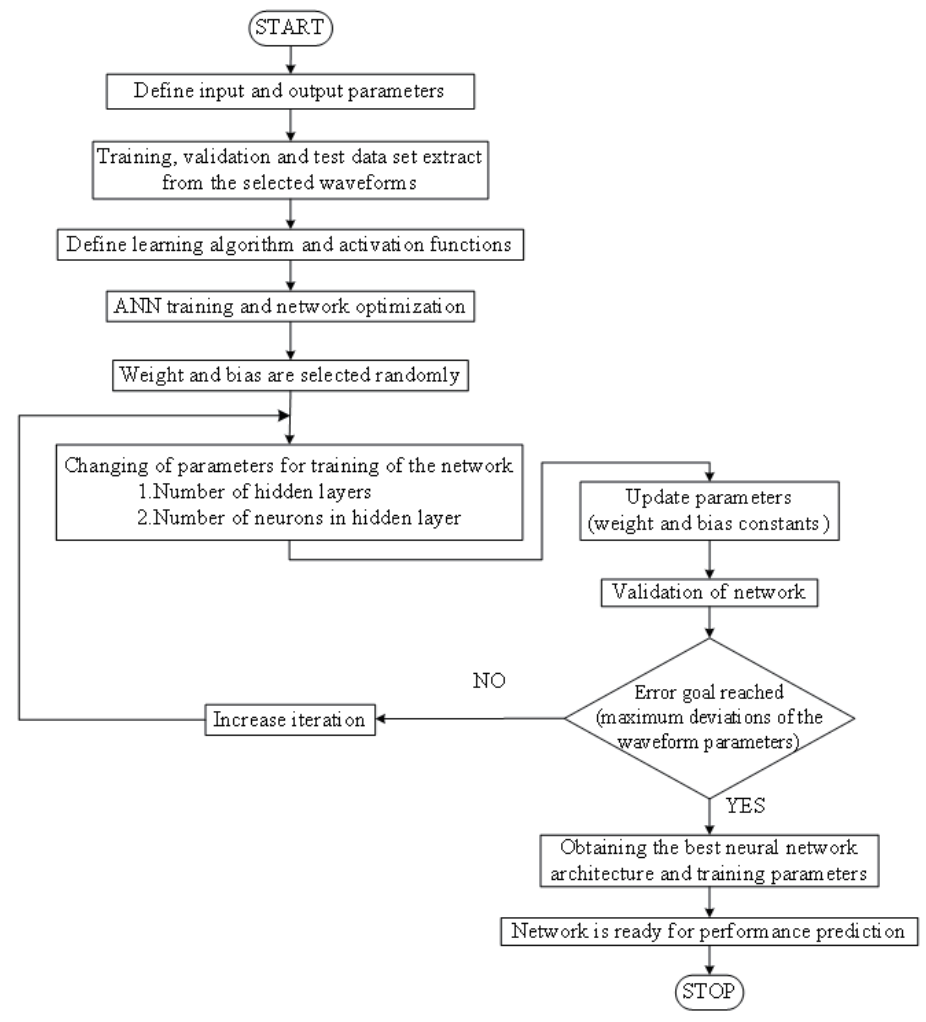

Fig. 3. Flow chart of evaluation of impulse voltage waveform.

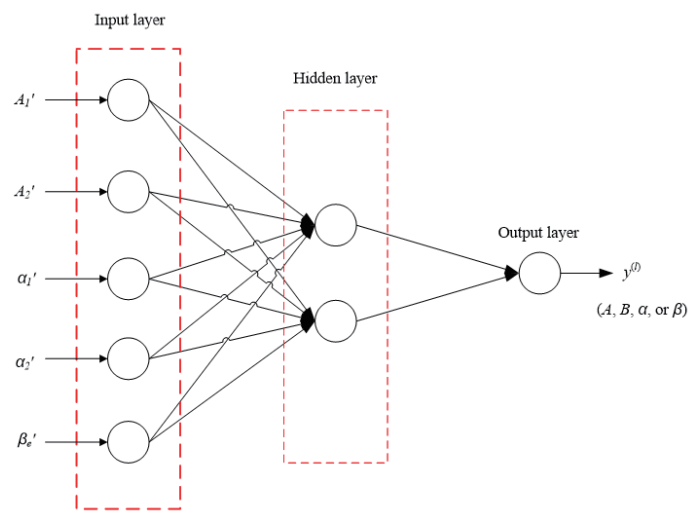

Fig. 4. (Color online) Architecture of ANN.

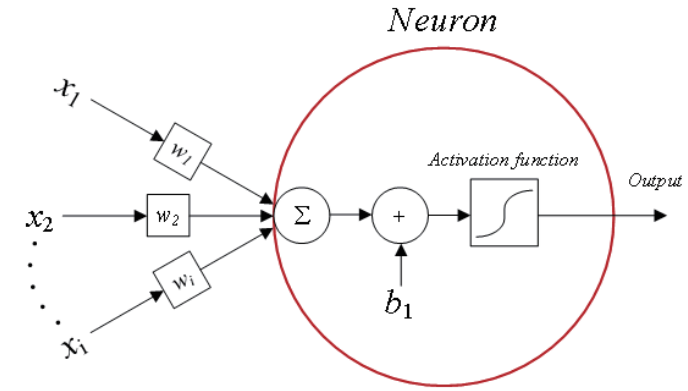

Fig. 5. (Color online) Network model of the neural network for evaluating the estimated base waveform.

parameter $i$ and neuron $j$ of the hidden layer, respectively. $w_{2, j}^{(l)}$ and $b_{2, j}^{(l)}$ are the weighting and bias parameters of the hidden layer associated with neuron $\mathrm{j}$ of the hidden layer, respectively. $f(\cdot)$ is the selected Tansig activation function given by Eq. (8). $n$ is the number of neurons in the hidden layer, so in this case, $n=2$.

$$
y^{(l)}=\sum_{j=1}^{n}\left\{w_{2, j}^{(l)} f\left(\sum_{i=1}^{N}\left(w_{1, i j}^{(l)} \cdot x_{i}+b_{1, i j}^{(l)}\right)\right)+b_{2, j}^{(l)}\right\}
$$




$$
f(x)=\frac{2}{1+e^{-2 x}}-1
$$

For convenience of calculation, Eq. (7) can be rewritten in matrix form as Eq. (9), where $X$ is the input vector expressed by Eq. (10). $w_{1}^{(l)}$ and $b_{1}^{(l)}$ are a weighting matrix and a bias vector of the input layer, respectively. $w_{2}^{(l)}$ and $b_{2}^{(l)}$ are a weighting matrix and a bias vector of the hidden layer, respectively.

$$
\begin{gathered}
y^{(l)}=w_{2}^{(l)}\left\{f\left(w_{1}^{(l)} X+b_{1}^{(l)}\right)\right\}+b_{2}^{(l)} \\
X=\left[\begin{array}{lllll}
A^{\prime} & B^{\prime} & \alpha^{\prime} & \beta^{\prime} & \beta_{e}^{\prime}
\end{array}\right]^{t}
\end{gathered}
$$

The values of $w_{1}^{(l)}, w_{2}^{(l)}, b_{1}^{(l)}$, and $b_{2}^{(l)}$ are given as Eqs. (11)-(14), respectively.

$$
\begin{aligned}
& \begin{array}{c}
\left(w_{1}^{(A)}\right)^{t}=\left[\begin{array}{cc}
2.638 & -8.428 \\
4.691 & -0.426 \\
33.071 \times 10^{-6} & 30.47 \times 10^{-6} \\
0.6715 \times 10^{-6} & -0.2314 \times 10^{-6} \\
-6.618 & -12.354
\end{array}\right], \quad\left(w_{1}^{(B)}\right)^{t}=\left[\begin{array}{cc}
-2.516 & -4.043 \\
-3.835 & -14.619 \\
12.313 \times 10^{-6} & -191.85 \times 10^{-6} \\
-0.01761 \times 10^{-6} & -0.07119 \times 10^{-6} \\
-2.639 & 3.639
\end{array}\right], \\
\left(w_{1}^{(a)}\right)^{t}=\left[\begin{array}{cc}
-6.592 & 0.026 \\
-1.472 & -0.002 \\
-188.967 \times 10^{-6} & 30.135 \times 10^{-6} \\
-0.5037 \times 10^{-6} & 0.00061 \times 10^{-6} \\
-7.248 & 0.007
\end{array}\right], \quad\left(w_{1}^{(\beta)}\right)^{t}=\left[\begin{array}{cc}
0.242 & 44.954 \\
0.019 & 14.909 \\
-2.454 \times 10^{-6} & 53.426 \times 10^{-6} \\
-0.1510 \times 10^{-6} & 0.6199 \times 10^{-6} \\
0.363 & 19.971
\end{array}\right]
\end{array} \\
& w_{2}^{(A)}=\left[\begin{array}{ll}
8.455 & 20.530
\end{array}\right] \times 10^{-2} \\
& w_{2}^{(B)}=\left[\begin{array}{ll}
-29.764 & -7.699
\end{array}\right] \times 10^{-2} \\
& w_{2}^{(\alpha)}=\left[\begin{array}{ll}
-0.350 & 3.322
\end{array}\right] \times 10^{4} \\
& w_{2}^{(\beta)}=\left[\begin{array}{ll}
-6.825 & -1.873
\end{array}\right] \times 10^{6} \\
& b_{1}^{(A)}=\left[\begin{array}{c}
-1.918 \\
8.845
\end{array}\right], \quad b_{1}^{(B)}=\left[\begin{array}{l}
-1.183 \\
-2.717
\end{array}\right], \\
& b_{1}^{(\alpha)}=\left[\begin{array}{c}
14.562 \\
-0.455
\end{array}\right], \quad b_{1}^{(\beta)}=\left[\begin{array}{c}
0.437 \\
-30.672
\end{array}\right]
\end{aligned}
$$




$$
\begin{aligned}
& b_{2}^{(A)}=1.041 \\
& b_{2}^{(B)}=-0.887 \\
& b_{2}^{(\alpha)}=1.764 \times 10^{4} \\
& b_{2}^{(\beta)}=6.097 \times 10^{6}
\end{aligned}
$$

Using the developed model in matrix form given in Eqs. (9) and (10), we can determine the base curve and its parameters. Then, procedures (3) to (6) are employed to evaluate the lightning impulse voltage parameters as the standard recommendation as presented in Sect. 1.

\section{Simulation Results}

The validity of the proposed method is verified using impulse voltage waveforms, i.e., 29 cases (LI-A1 to LI-A12 and LI-M1 to LI-M17) collected from the TDG program ${ }^{(18)}$ and two experimental waveforms (X1 and X2) with different overshoot rates. The experimental waveforms $\mathrm{X} 1$ and $\mathrm{X} 2$ have high damped oscillation around the peak with a frequency of about $250 \mathrm{kHz}$ and overshoot rates of 14.8 and 23\%, respectively. Examples of the waveforms evaluated by the proposed method are shown in Figs. 6-11. Solid black lines show the recorded waveforms

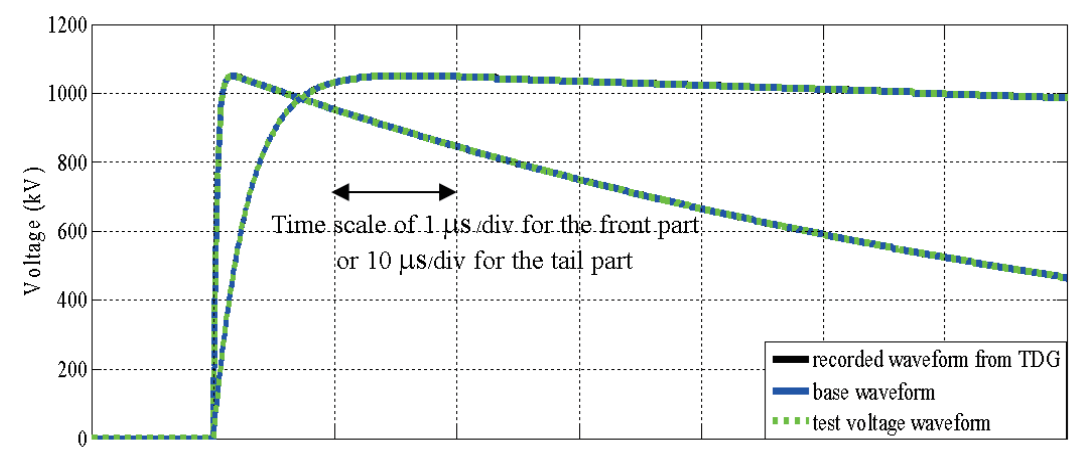

Fig. 6. (Color online) Evaluated waveforms in the case of LI-A1.

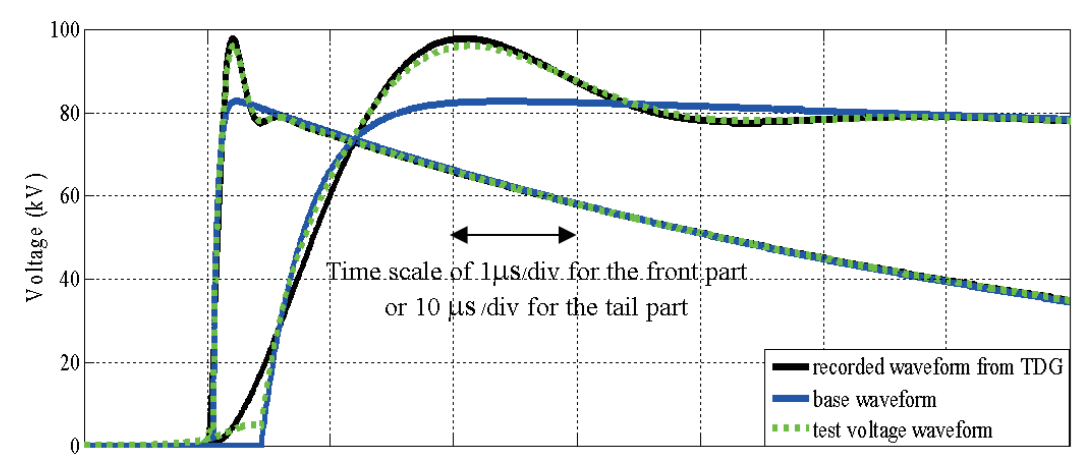

Fig. 7. (Color online) Evaluated waveforms in the case of LI-A8. 




Fig. 8. (Color online) Evaluated waveforms in the case of LI-A11.

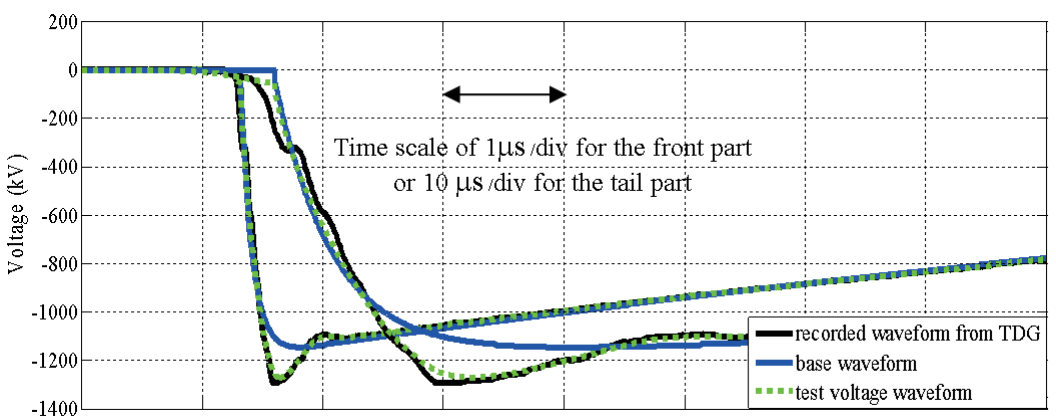

Fig. 9. (Color online) Evaluated waveforms in the case of LI-M7.



Fig. 10. (Color online) Evaluated waveforms in the case of LI-M8.



Fig. 11. (Color online) Evaluated waveforms in the case of LI-M15. 
from the TDG program, solid blue lines represent the base waveforms evaluated by the proposed method, and green dotted lines represent the test waveforms computed by the proposed method. The evaluated waveform parameters, i.e., the peak voltage $\left(U_{p}\right)$, the front time $\left(T_{1}\right)$, the time to half $\left(T_{2}\right)$, and the overshoot rate $\left(\beta_{e}\right)$, and their deviation from the standard values, are presented in Fig. 12. By comparing the results evaluated by the proposed method and the standard values,

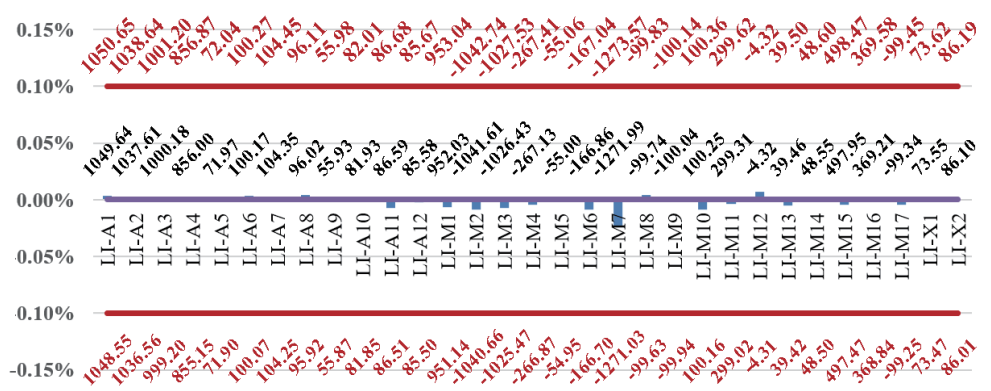

(a)



(b)

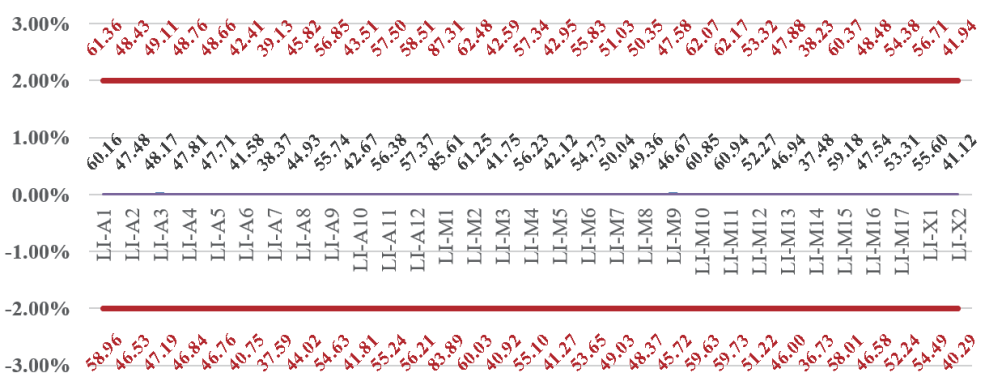

(c)



(d)

Fig. 12. (Color online) Waveform parameters and their deviations assessed by the proposed method in comparison with the acceptance limits of the standard. (a) Peak voltage $\left(U_{p}\right)$ in $\mathrm{kV}$, (b) front time $\left(T_{1}\right)$ in $\mu \mathrm{s}$, (c) time to half $\left(T_{2}\right)$ in $\mu \mathrm{s}$, and (d) overshoot rate $\left(\beta_{e}\right)$ in percent. 
it can be seen that all results are within acceptable limits. From Fig. 12(a), it can be seen that the highest error of the peak voltage $\left(U_{p}\right)$ is only $0.02 \%$ (the acceptable tolerance is $0.1 \%$ ) in the case of LI-M7. The highest error of the front time $\left(T_{1}\right)$ in Fig. 12(b) is $1.59 \%$ for the case of LI-M12, which is still less than the acceptable tolerance of $2.0 \%$. In addition, Fig. 12(c) shows that the highest error of the time to half $\left(T_{2}\right)$ is only $0.05 \%$ (the acceptable tolerance is $0.1 \%$ ), which is for the case of LI-A3. As shown in Fig. 12(d), the highest error of the overshoot rate $\left(\beta_{e}\right)$ is only $0.08 \%$, which is for case LI-A5, compared with the acceptable tolerance of $1.0 \%$. Note that the errors of the waveform parameters computed by the proposed method are due to the vertical resolution and sampling frequency used in this paper differing from those used by the standard. However, with the 12-bit resolution and $100 \mathrm{MHz}$ sampling frequency used in this paper, the method is still very accurate, and all errors are still within the acceptable tolerances according to the standard.

\section{Conclusion}

An effective approach based on a neural network method for evaluating the lightning impulse voltage waveform parameters has been proposed in this paper. The ANN was developed by a back-propagation algorithm as pre-calculation using 29 waveform data collected from the standard. Simple estimated parameters were proposed and utilized as the input parameters. The developed ANN was transformed into matrix form, making it very convenient to use in correcting the base curve. From the simulation results in all cases (29 cases from the standard and two experimental cases), it was confirmed that the proposed approach can provide good accuracy in waveform parameter evaluation. The highest errors of the peak voltage $\left(U_{p}\right)$, front time $\left(T_{1}\right)$, time to half $\left(T_{2}\right)$, and overshoot rate $\left(\beta_{e}\right)$ evaluated by the proposed method were 0.02 , $1.59,0.05$, and $0.08 \%$, respectively. In addition, the proposed method provides better performance in terms of execution time than the standard recommended method (Levenberg-Marquardt) due to no requirement of an iteration process. It can be concluded that the proposed method is an attractive choice for the waveform parameter evaluation of lightning impulse voltages.

\section{Acknowledgments}

The authors would like to give special acknowledgement to the School of Engineering, King Mongkut's Institute of Technology Ladkrabang for providing the facility in this research. This project was funded by National Research Council of Thailand.

\section{References}

1 IEC Standard 60060-1:2010 (2010) 1.

2 IEEE Standard 4-2013 (Revision of IEEE Std 4-1995) (2013) 1.

3 P. L. Lewin, T. N. Tran, D. J.Swaffield, and J. K. Hällström: IEEE Trans. Power Delivery 23 (2010) 3. https:// doi.org/10.1109/TPWRD.2007.911124

4 P. Simon, F. Garnacho, S. M. Berlijn, and E. Gockenbach: IEEE Trans. Power Delivery 21 (2006) 560. https:// doi.org/10.1109/TPWRD.2005.858807

5 M. Hinow, W. Hauschild, and E. Gockenbach: IEEE Trans. Dielectr. Electr. Insul. 17 (2010) 1628. https://oi. org/10.1109/TDEI.2010.5595567 
6 S. Okabe, T. Tsuboi, G. Ueta, J. Takami, and H. Hirose: IEEE Trans. Dielectr. Electr. Insul. 17 (2010) 2. https:// doi.org/10.1109/TDEI.2010.5411995

7 G. Ueta, T. Tsuboi, and S. Okabe: IEEE Trans. Dielectr. Electr. Insul. 18 (2011) 783. https://doi.org/10.1109/ TDEI.2011.5931066

8 G. Ueta, T. Tsuboi, and S. Okabe: IEEE Trans. Dielectr. Electr. Insul. 19 (2012) 352. https://doi.org/10.1109/ TDEI.2012.6148538

9 C. R. H. Barbosa, M. T. F. Silva, L. C. Azevedo, and L. C. Faria: IEEE Trans. Instrum. Meas. 64 (2015) 1378. https://doi.org/10.1109/TIM.2015.2406059

10 Y. M. Li, J. Kuffel, and W. Janischewskyj: IEEE Trans. Power Delivery 8 (1993) 1727. https://doi. org/10.1109/61.248280

11 S. Okabe, T. Tsuboi, and J. Takami: IEEE Trans. Dielectr. Electr. Insul. 16 (2009) 1124. https://doi.org/10.1109/ TDEI.2009.5211865

12 J. Yang, Q. Zhou, C. Qu, and Hai-ping Hou: 2012 IEEE 11th Int. Conf. Signal Processing (2012) 856-860.

13 S. Venkatesan, P. Vanaja Ranjan, and D. Ashokaraju: Conf. Convergent Technologies for Asia-Pacific Region (TENCON, 2003) 1562-1566.

14 S. Roychowdhury: 1998 IEEE Int. Conf. Systems, Man, and Cybernetics (SMC'98, 1998) 4022-4027.

15 S. Sato and M. Sasaki: 2007 Int. Power Engineering Conf. (IPEC, 2007) 1278-1281.

16 N. Pattanadech and P. Yutthagowith: IEEE Trans. Dielectr. Electr. Insul. 22 (2015) 2931. https://doi.org/10.1109/ TDEI.2015.005165

17 P. Yutthagowith and N. Pattanadech: IEEE Trans. Power Delivery 31 (2016) 271. https://doi.org/10.1109/ TPWRD.2015.2448640

18 IEC Standard 61083-2:2013(2013) 1.

19 A. Delaunoy, A. Wehenkel, T. Hinderer, S. Nissanke, C. Weniger, A. R. Williamson, and G. Louppe: 3rd Workshop on Machine Learning and the Physical Sciences (NeurIPS, 2020) 1-8.

20 K. T. M. U. Hemapala, O. V. G. Swathika, and K. P. R. D. S. K. Dharmadasa: Develop. Eng. 3 (2018) 100. https://doi.org/10.1016/j.deveng.2018.05.003

21 S. Prakash, N. P. Gopinath, and B. Kalaiselvi: Int. J. Innov. Technol. Explor. Eng. (IJITEE, 2019) 604. https:// doi.org/10.35940/ijitee.B1189.1292S419

\section{About the Authors}

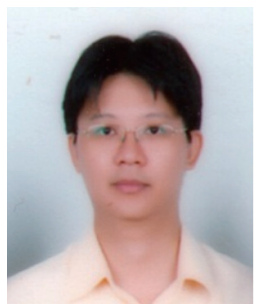

Nutthaphong Tanthanuch received his B.Eng., M.Eng., and Ph.D. degrees from Chulalongkorn University, Bangkok, Thailand, in 2001, 2004, and 2011, respectively. He joined Thammasat University as a lecturer in 2009. His research interests are in gas discharge, electric field calculation, and condition monitoring of high-voltage equipment.



Savinee Ludpa received her B.Eng. and M.Eng. degrees from King Mongkut's Institute of Technology Ladkrabang, Bangkok, Thailand, in 2006 and 2009, respectively, and she is currently studying for her Ph.D. degree at King Mongkut's Institute of Technology Ladkrabang while working at Metropolitan Waterworks Authority as an engineer. Her research interests are in highvoltage engineering. 


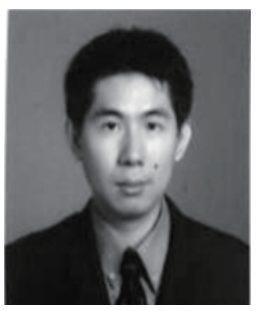

Peerawut Yutthagowith received his B.Eng. and M.Eng. degrees from Chulalongkorn University, Bangkok, Thailand, in 1998 and 2002, respectively, and his Ph.D. degree from Doshisha University, Kyoto, Japan, in 2010. He joined King Mongkut's Institute of Technology Ladkrabang as a lecturer in 2007. His research interests are in high-voltage engineering and electromagnetic transients in power systems. Dr. Yutthagowith is also a member of the International Council on Large Electric Systems (CIGRE)WG C4.37. 